Extending the Farkas Lemma Approach

to Necessity Conditions

to Infinite Programming

R.A. Tapia

M.W. Trosset

September, 1991

(revised May 5, 1993)

TR.91-25 



\title{
Extending the Farkas Lemma Approach to Necessity Conditions to Infinite Programming
}

\author{
R.A. Tapia * M.W. Trosset ${ }^{\dagger}$ \\ May 5, 1993
}

*Department of Computational and Applied Mathematics and Center for Research in Parallel Computation, Rice University, Honston, Texas 77251-1892. This author was supported in part by NSF (oop. Agr. No. (! (R-8809615, AFOSR 89-0363, DOE DEFG0586ER25017 and ARO 9DAAL03-90)-(i-0)(093.

${ }^{\dagger}$ Department of Computational and Applied Mathematics (Adjunct), Rice University; Department of Psychology (Adjunct), University of Arizona; and Consultant, P.O. Box 40993, Tucson, Arizona 85717-(0993. This author was supported in part by NSF Coop. Agr. No. CCR-8809615 as a visiting member of the Center for Research in Parallel Computation, Rice University, Houston, Texas 77251-1892, July 1991, October 1992, and Spring 1993. 



\begin{abstract}
Under mild assumptions, the classical Farkas lemma approach to Lagrange multiplier theory is extended to an infinite programming formulation. The main result generalizes the usual first-order necessity conditions to address problems in which the domain of the objective function is Hilbert space and the number of constraints is arbitrary. The result is used to obtain necessity conditions for a well-known problem from the statistical literature on probability density estimation.
\end{abstract}

Key words: Lagrange multiplier theory, Farkas lemma, infinite programming, mathematical progranming. 



\section{Introduction}

In 1951, Kuhn and Tucker [14] developed a Lagrange multiplier theory for mathematical programming problems that contain inequality constraints. In this theory, the domain of the objective function is Euclidean space and the constraint functionals are indexed by a finite set. Their development, explicated and popularized by Fiacco and McCormick ([6], Chapter 2), invokes the classical Farkas lemma (Farkas [5]) to generate a vector (the Lagrange multipliers) that can be viewed as a "weighting" of the finite set of constraints. For years we lave tanght this material, each time pondering the extent to which this development of Lagrange multiplier theory depends on the finite dimensionality of Enclidean space aud the finiteness of the constraint set. Somewhat recently, our interest was enhanced when we learned of an interesting infinite programming problem in the statistics literature for which we could state a formal generalization of the usual first-order necessity conditions, with no known theoretical justification for doing so. In the present study of Lagrange 1multiplier theory, we have not only succeeded in generalizing the Farkas lemma approach, but have also acquired new insight into the essential features of that approach.

Of late, it has become fashional,le to refer to the first-order necessity conditions as the "Karush-Kuhn-Tucker conditions," rather than the "KulınTucker conditions." This observation motivated us to carefully study the origins of these conditions, resulting in a fascinating excursion into the history of nonlinear programming and the classical calculus of variations. Because of the pedagogical nature of the present work, we believe that it is appropriate to share what we have learned. In passing, we note that Prékopa [17] effectively argues that fundanental irleas concerning the optimality conditions for nonlinear programming can be found in some early papers in mechanics by Fourier, Cournot, and Farkas and also by Gauss, Ostrogradsky and Hamel.

In the 1930s, there flomished at the University of Chicago a school of thought in the calculus of variations that was founded by C. A. Bliss. Researchers associated with this school inchuded L. M. Graves, H. H. Goldstine, M. R. Hestenes, A. S. Howseholeler, W. Karush, E. J. Mcshane, W. T. Reid, F. A. Valentine, and many others. Lagrange multiplier theory for the equality constrained mathematical programming problem was known, in one form or another, to most researchers in the classical calculus of variations, who were fully aware that it conld be derived from varions theories for more general 
problems. Bliss [3] presented an elegant exposition of this theory in the first section of a 1938 survey of normality and almormality in the calculus of variations. The first section of Bliss's article is entitled, "Abnormality for minima of functions of a finite number of variables," and it begins, "The significance of the notion of abnommality in the calculus of variations can be indicated by a study of the theory of the simpler problem of finding ..." (p. 367). What is particularly enlightening about this section is that it reveals how the Chicago school regarded finite-dimensional problems: in a pre-computational era, the theoretically less challenging case of finite dimensions was primarily valued as a training ground for developing intuition about more difficult problems.

Another interest of the (hicago school was the incorporation of inequality constraints. They often used squared slack variables to extend known theory and insight from the more standard equality constrained problem to the less standard inequality constrained problem. Hestenes recalled that this device, as well as the techuigues of successive linear and quadratic programming (techniques usually attributed to the post-war mathematical programming community), was a standard tool of the ('hicago school.

In his $1937 \mathrm{Ph} . \mathrm{D}$. thesis, Valentine [23] studied the problem of Lagrange with differential inequality constraints of the form

$$
y(x, y, d y / d x) \geq 0
$$

He replaced this constraint with the equality const,raint

$$
g(x, y, d y / d x)=(d z / d x)^{2}
$$

where $z(x)$ is an auxiliary function satisfying a particular initial condition, and applied the standard theory. Today, many anthors refer to the use of squared slack variables as the "Method of Valentine." The first anthor of the present paper, undoubtedly iufluenced by the instruction of Hestenes and Valentine in his graduate education at UCLA, often employs this method to develop insight in elementary conses. For example, a quick way to derive Lagrange multiplier theory for general nonlinear programming is to add squared slack variables to the inequality constraints, then apply the standard theory for equality constrained nonlinear programming. This approach does not establish the nomnegativity of the multipliers of the inequality constraints; however, their nommegativity follows directly from the second-order 
necessity conditions. Collectively, then, one obtains the same first-order and second-order necessity conditions from this elementary approach. The price that one pays is that regularity (linear independence of the gradients of the binding constraints) must be assunned, so that it is not possible to reap the benefits of more subtle constraint qualifications.

Given these interests of the ('hicago school, it made perfect sense for Graves to assign to Karusl, as a topic suitable for his master's thesis, the simple problem of extending. Bliss's finite-dimensional treatment of equality constraints to the case of inequality constraints. Karush [12] handled his assignment beautifully, leriving first-order necessity conditions using the Farkas lemma and formally stating the Kulun-Tucker constraint qualification as "Property Q." Thus, Karush's 1939) thesis contains the Kuhn-Tucker theory in all of its particulars. The constraint qualification Q was implicit in Bliss [3], who slowed that it was implied by regularity, which he then assumed. Karush, however, assumed only the constraint qualification, according it a privileged status that Bliss had not.

One characteristic of the wswal (Kulnu-Tucker) approach to Lagrange multiplier theory is that it repuires the multiplier of the gradient of the objective function (say $\lambda_{0}$ ) to be unity. This requirement is what necessitates a constraint qualification: it is generally known that this hypothesis is unnecessary if $\lambda_{0}$ is allowed to vary freely. For nonlinear programming with inequality constraints, this fact is usually attriluted to Jolnn [11] and called the Fritz Joln condition. (The device itself was first studlied by Mayer [15]; in fact, this is the standard formulation of Lagrange multiplier theory in the calculus of variations.) It is of value, here, to point out that Karush also presented a form of the John theory. A straightforward application of Bliss's treatment of the equality constrained problem to the squared slack variable formulation of the inequality constrained problem led him to a result that is identical to John's, but without the sign restrictions on the nultipliers. Karush then observed that the sign restrictions followed from Bliss's second-order necessity theory. As we have already olserved, however, the price of this derivation is the assumption of regularity and two continuous clerivatives. Accordingly, Karusl employed this approach in a manner that we have attributed to the Chicago school, viz. to olstain insiglits that, led lim to the Karush-KuhnTucker theory.

Karush was never enconaged to publish his work, presumably because the finite-dimensional case was then deemed too elementary to be of inde- 
pendent interest. It remained virtually unkuown until Kulın discovered a reference to it in a 1974 textbook by Takayama [19] and obtained a copy of the thesis. Kuhn attempted to "set the record straight" at a 1975 AMS symposium (Kuln [13]), and even went so far as to refer to the "Karush conditions." The same year, Hestenes [10], who had directed Karush's Ph.D. thesis, noted Karush's work in lis book on optimization theory. Even so, it is only very recently that Karnsh's work has been generally acknowledged.

In summary, our historical investigations lave led us to strongly support the "Karush-Kuhn-Tucker" terminology. There is no question of the importance of the Kuhn-Tucker paper in the history of mathematical programming, but there is also no question that Karush obtained the identical result twelve years earlier. Furthermore, not only is Karush himself deserving of recognition, but we believe that the nse of his name is a fitting tribute to the members of the great (hicago school of Bliss, whose deep understanding of mathematical programming has not been properly recognized and appreciated. Becanse it was not nuti] the 1950) that there was a clemand for the finite-dimensional theory, the (hicago researchers were simply two decades ahead of their time. The Karush-Kuln-Tucker conditions represent a rare instance in which it is possible to document just how much of mathematical programming these researchers understood and anticipated.

Returning to the present paper, our objective is to extend the classical Farkas lemma approach to nuathenatical progranming problems in which the domain of the objective function is Hilbert space and the constraint functionals are indexed by an arbitrary set. Onr approach carefully mimics the finite programming development. It is based on a generalized Farkas lemma, and replaces the Lagrange multiplier vector with a measure on the (possibly infinite) index set. If this measure is absolutely continuous, then it can be represented as a (density) function on the index set. Because our point of view may seem unnatual to some readers otherwise familiar with Lagrange multiplier theory, we briefly digress to motivate it.

Consider vectors $x_{1}, \ldots, x_{k} \in \mathrm{R}^{n}$, scalar weights $u_{1}, \ldots, u_{k} \in \mathrm{R}^{n}$, and the weighted sum

$$
\sum_{i \in l} u_{i} r_{i}
$$

where the index set $I=\{1, \ldots, k\}$. By defining a measure $\mu$ on the Borel sets of $\mathrm{R}^{n}$ that concentrates on $\left\{r_{1}, \ldots, x_{k}\right\}$ and satisfies $\mu\left(\left\{x_{i}\right\}\right)=u_{i}$, we 
can write

$$
\sum_{i \in I} u_{i} u_{i}=\int_{\mathrm{R}^{n}} x \mu(d x) .
$$

Thus, a set of weights can be viewed as a measure and a weighted sum can be viewed as a (Lebesgue) integral with respect to that measure. When the weights are nomnegative and smm to mity, $\mu$ is a probability measure and probabilists call the integral an expectation.

Now consider the index nap $i \mapsto x_{i}$, which embeds $I$ in $\mathrm{R}^{n}$. The measure $\mu$ induces a measure $u$ on the subsets of $I$ by $u(\{i\})=\mu\left(\left\{x_{i}\right\}\right)$. This allows us to further write

$$
\sum_{i \in I} u_{i} x_{i}=\int_{R^{u}} x \mu(d x)=\int_{I} x_{i} u(d i) ;
$$

hence, our point of view that a set of weights is a measure on an inclex set. It is this perspective that will lear to a manageable statement of generalized first-order conditions.

The flavor of our generalization of Lagrange multiplier theory is not entirely new. Semi-infinite programming is also concerned with problems in which the constraint functionals are indexed by an infinite set, although the domain of the objective function is still assinned to be Eucliclean space. The famous paper by John [11] posed a seni-infinite programming problem; however, John exploited the finite dimensionality of $K^{n}$ to reduce the number of constraints to $n+1$. More urently, a multiplier theorem of precisely the sort that we seek was oltained by Colserna, López, and Pastor [8]. The authors use a generalized Fartas lemma and retain the full set of constraints; however, their result also dependis critically on the finite dimensionality of Euclidean space.

It should be noted that a munber of anthors have published multiplier theorems in very abstract settings. The standard formulation is that of Guignard [9], who derived both necessity and sufficiency conditions for the problem

$$
\begin{aligned}
\text { maximize } & \phi(x) \\
\text { subject to } & x \in C \subset X \\
& a(x) \in B \subset Y,
\end{aligned}
$$

where $X$ and $Y$ are real Banach spaces and $\psi: X \rightarrow(-\infty,+\infty)$ and $a$ : $X \rightarrow Y$ are Fréchet differentiahle. Cnignard's multiplier is an element of the 
topological dual space of $Y$, and her entire approach is markedly different from ours.

The primary purpose of the present paper is pedagogical. That is, we wish to demonstrate that by (i) interpreting the vector of Lagrange multipliers as a measure on the index set of constraints and by (ii) utilizing tools from functional analysis and probability theory, the standard finite-dimensional approach to multiplier theory (Karnsl-Kulnu-Tucker) can be successfully generalized to infinite progranming in Hilbert space. This exercise, however, is not entirely pedagogical, for we also believe that there are important infinite programming problems to which on theory can be profitably applied. Therefore, after in Section 2 deriving first-order necessity conditions for general infinite programming problens, in Section 3 we will consider results that facilitate the use of these conditions. In Section 4, by way of an example, we will also apply this theory to oldain necessity conditions for a constrained optimization problem from the statistical literature on probability density estimation. However, we lave deferred to another paper (Trosset [21]) an investigation of the statistical conseruences of these conditions.

\section{Main Theorem}

We begin with a real Hilhert space $X$ with imner product $\langle\cdot, \cdot\rangle$. By the general nonlinear programming prohlen - problem (NLP) for short - we mean the constrained optinization problem

$$
\begin{array}{cl}
\text { maximize } & f(x) \\
\text { subject to } & g_{\alpha x}(x) \geq 0 \quad \forall \alpha \in I \\
& h_{, \beta}(x)=0 \quad \forall \beta \in I,
\end{array}
$$

where $f, g_{\alpha}, h_{\beta}: X \rightarrow(-\infty,+\infty)$. We assume that the index sets $I$ and $I$ have corresponding sigma fields $\mathbf{I}$ and $\mathbf{J}$ such that the pairs $(I, \mathbf{I})$ and $(J, \mathbf{J})$ are measure spaces. Measures on these spaces will be denoted by $t, u, \lambda$, etc. At times, we will also endow $I$ and $I$ with topologies. Typically, $I$ and $J$ will be subsets of Enclidean space. For each $x \in X$, we define the index subset $I_{0}(x):=\left\{\alpha \in I: g_{c r}(x)=0\right\}$.

We assume that $f, g_{c}, h_{\beta} \in C^{1}(X)$. For each $x \in X$, the sets $\nabla A_{0}(x):=$ $\left\{\nabla g_{\alpha}(x): \alpha \in I_{0}(x)\right\}$ and $\nabla B(x):=\left\{\nabla h_{\beta}(x): \beta \in . I\right\}$ are assumed to be 
Borel measurable. We also assinue that the index maps $\alpha \mapsto \nabla g_{\alpha}(x)$ and $\beta \mapsto \nabla h_{\beta}(x)$ are Borel binneasuralle functions. This will enable measures on $I$ and $J$ to induce measures on $X$, and also conversely. Measures on $X$ will be denoted $\nabla F, \nabla G$, etc.

For technical reasons, we will sonetinnes further assume that the functions $g_{\alpha}$ and $h_{\beta}$ are elements of a real Hillert space $\Gamma$. In that event, we will assume that the sets $A_{0}(x):=\left\{\mathscr{y}_{c}: \alpha \in I_{0}(x)\right\}$ and $B:=\left\{h_{\beta 1}: \beta \in J\right\}$ are Borel measurable. We will also assunne that the index maps $\alpha \mapsto g_{c}$ and $\beta \mapsto h_{\beta}$ are Borel measurable functions. This will enable measures on $I$ and $J$ to induce measures on $\Gamma$. Such measures will be denoted by $F$, $G$, etc.

In this section we will alerive necessary conditions for a point $x^{*} \in X$ to be a local solution of problem (NLP). To do so, we modify and generalize Fiacco's and McCormick's [G] presentation of the first-order theory for the finite-dimensional case. The key to this generalization is the concept of the expectation of a measure on a IIillert space. Toward this end, in what follows $H$ will denote a real Hillsert space with inner product $(\cdot, \cdot)$. Following Parthasarathy ([16], Definition 3.2, 1) 168) we make the following definition.

Definition 2.1 Let $\mu$ be a merasure on $H$. If the linear functional $L(y):=$ $\int(y, x) \mu(d x)$ is continuous, then the expectation of $\mu$, which we denote by $\int x \mu(d x)$, is defined to be the Rirs: representer of $L$.

At this point it will be of value to introduce some basic notation. Let $\mathbf{M}(K)$ denote the fanily of totally finite positive measures that concentrate on the set $K \subset H$, and let $\mathrm{M}_{1}(K)$ denote the fanily of probability measures that concentrate on the set $K \subset H$. We are interested in the sets of expectations

$$
C\left(K^{\prime}\right)=\left\{\int x \mu(d x): \mu \in \mathbf{M}(K)\right\}
$$

and

$$
C_{1}(K)=\left\{\int x \mu(d x): \mu \in \mathrm{M}_{1}(K)\right\} .
$$

The set $C_{1}(K)$ is essentially the convex hull of $K$, and the set $C(K)$ is essentially the cone generated by $C_{1}\left(K^{\prime}\right)$. It should be clear that $C(K)$ and $C_{1}(K)$ are convex. In the next section we will demonstrate that $C_{1}(K)$ is also compact. The closedness of $\left(C /{ }^{\prime}\right)$ will be of fundamental importance in our theory. In the next section we will construct a condition which guarantees 
that $C(K)$ is closed. However, for the moment we will assume that it is closed.

We now generalize a fannous result.

Lemma 2.1 (Generalized Fulkus Lemma): Let $H$ denote a real Hilbert space with inner product $(\cdot, \cdot)$. Let $x_{0} \in H$ and $K \subset H$. Assume that $C(K)$ is closed. Then the following ait cruivulent:

(i) $\forall y \in H, \quad(y, x) \geq 0 \quad \forall x \in K$ entails $\left(y, x_{0}\right) \geq 0$;

(ii) $\exists \mu \in \mathbf{M}(K)$ such that $x_{0}=\int x \mu(d x)$.

Proof: We utilize the notion of a dual cone, introduced by Dieudonné [4] in his proof of the Halnu-Banach theorem. The dual cone $C^{*}$ of a cone $C$ is the set of all continuous linear finctionals nonnegative on $C$.

Consider (i). If $(y, x) \geq 0 \forall x \in K$, then $\left(y, \int x \mu(d x)\right)=\int(y, x) \mu(d x) \geq$ $0 \forall \mu \in \mathbf{M}(K)$, i.e. $y \in C\left(K^{\prime}\right)^{*}$. Hence, (i) is equivalent to the assertion that $y \in C(K)^{*}$ entails $\left(y, x_{0}\right) \geq 0 \forall y \in H$, or simply that $x_{0} \in C(K)^{* *}$. By Lemma 5.6 in Girsanov [7], $\left(K^{\prime}\right)^{* *}$ is the weak closure of the convex hull of $C(K)$. Since $C(K)$ is closed and convex, it follows that (i) is equivalent to $x_{0} \in C(K)^{* *}=C(K)$. But (ii) is a direct statement that $x_{0} \in C(K)$; hence, (i) and (ii) are equivalent.

Remark: It is possible to give an elenentary, but more complicated proof of this result. The very elegant proof that we have presented was suggested to us anonymously by the referee. This is an amusing realization of Valentine's [22] admonition to "always look at the dual situation when working with convex sets for it may save you some embarrassment."

Associated with problem (NI,P) is the generalized Lagrangian gradient

$$
\ell^{\prime}(x, u, \lambda):=\nabla_{x} f(x)-\int_{I} \nabla_{x} y_{\alpha}(x) u(d \alpha)+\int_{J} \nabla_{x} h_{\beta}(x) \lambda(d \beta),
$$

which is guaranteed to exist if the sets $\nabla A(x)$ and $\nabla B(x)$ are compact and the measures $u$ and $\lambda$ are totally finite. Our goal is to derive necessary conditions for solving problen (NLP) that involve this expression. We are now in a position to characterize some of these conditions.

Suppose that $x$ is a feasible point of problem (NLP). Let

$$
\begin{aligned}
Z_{1}(x):=\left\{z \in X:\left\langle z, \nabla y_{10}(x)\right\rangle\right. & \geq 0 \forall \alpha \in I_{0}(x), \\
\left\langle z, \nabla l_{h, \beta}(x)\right\rangle & =0 \forall \beta \in J, \quad\langle z, \quad \nabla f(x)\rangle \geq 0\},
\end{aligned}
$$




$$
\begin{aligned}
Z_{2}(x):=\left\{z \in X:\left\langle z, \nabla y_{i x}(x)\right\rangle \geq 0 \quad \forall \alpha\right. & \in I_{10}(x), \\
\left\langle z, \nabla h_{1 \beta}(x)\right\rangle & =0 \quad \forall \beta \in J, \quad\langle z, \quad \nabla f(x)\rangle<0\},
\end{aligned}
$$

Proposition 2.1 Let $K=\nabla A_{01}\left(x^{*}\right) \cup \nabla B\left(x^{*}\right)$. Assume

$A 1: \quad K$ is compact;

$A 2: \quad C(K)$ is closert.

If $x^{*}$ is a feasible point of problt.m. (NLP), then the following are equinalent:

(i) $Z_{2}\left(x^{*}\right)=\phi$.

(ii) There exist totally finit measures $u^{*}$ on $(I, \mathbf{I})$ and $\lambda^{*}$ on $(J, \mathbf{J})$ such that

(a) $\ell^{\prime}\left(x^{*}, u^{*}, \lambda^{*}\right)=0$,

(b) $g_{c r}\left(x^{*}\right) \geq 0 \quad \forall \quad \alpha \in I$,

(c) $h_{\beta}\left(x^{*}\right)=0 \forall \beta \in J$,

(d) $u^{*}\left(I^{\prime}\right)=0 \forall I^{\prime}$ mecusuruble: $\subset I \sim I_{0}\left(x^{*}\right)$,

(c) $u^{*}\left(I^{\prime}\right) \geq 0 \forall I^{\prime}$ me:usuruble: $\subset I$.

We will refer to the conditions (a)-(e) in (ii) as the generalized first-order conditions.

Proof: Assume (ii) and sulpose that $z \in Z_{2}\left(x^{*}\right)$. Then

$$
\begin{aligned}
0 & >\left\langle z, \nabla f\left(x^{*}\right)\right\rangle \\
& \left.=\left\langle z, \int_{I} \nabla g_{c x}\left(x^{*}\right) u^{*}\left(d u^{\prime}\right)-\int_{J} \nabla h_{\beta \beta}\left(x^{*}\right)\right\rangle \lambda^{*}(d l \beta)\right\rangle \\
& =\int_{I}\left\langle z, \nabla g_{u}\left(x^{*}\right)\right\rangle u^{*}(d \alpha)-\int_{J}\left\langle z, \nabla h_{i \beta}\left(x^{*}\right)\right\rangle \lambda^{*}(d \beta) \\
& \geq 0,
\end{aligned}
$$

which is a contradiction. This proves that (ii) implies (i).

Conversely, suppose that $Z_{2}\left(x^{*}\right)=\phi$. Then, if $z$ satisfies

$$
\begin{array}{ll}
\left\langle z, \nabla g_{c v}\left(x^{*}\right)\right\rangle \geq 0 & \forall \alpha \in I_{0}\left(x^{*}\right) \\
\left\langle z, \nabla h_{\beta \beta}\left(x^{*}\right)\right\rangle \geq 0 & \forall \beta \in . J \\
\left\langle z,-\nabla h_{\beta \gamma}\left(x^{*}\right)\right\rangle \geq 0 & \forall \beta \in J,
\end{array}
$$


$z$ must also satisfy $\left\langle z, \nabla f\left(x^{*}\right)\right\rangle \geq 0$. But this implication is (i) in Lemma 2.1 , so we may conclude that

$$
\begin{aligned}
\nabla f\left(x^{*}\right) & =\int_{\nabla A_{0}\left(x^{*}\right)} y \nabla F_{0}(d ! y)+\int_{\nabla B\left(x^{*}\right)} y \nabla F^{\prime}(d y)-\int_{\nabla B\left(x^{*}\right)} y \nabla F^{\prime \prime}(d y) \\
& =\int_{I_{0}\left(x^{*}\right)} \nabla g_{k v}\left(x^{*}\right) l_{l_{1}}(d \gamma)+\int_{J} \nabla h_{i \beta}\left(x^{*}\right) t^{\prime}(d \beta)-\int_{J} \nabla h_{\beta}\left(x^{*}\right) t^{\prime \prime}(d \beta) .
\end{aligned}
$$

We now obtain conditions (a)-(e) ly setting $u^{*}=t_{0}$ on $I_{0}\left(x^{*}\right), u^{*}=0$ on $I \sim I_{0}\left(x^{*}\right)$, and $\lambda^{*}=-\left(t^{\prime}-t^{\prime \prime}\right)$ on $J$.

Our statement of first-order condition (a) is somewhat nontraditional. Suppose that the $g_{c}$ and $h_{\beta}$ are elencents of a real Hilbert space $\Gamma$. Assuming that the indicated expectations cxist (which, of course, they may not), define the generalized Lagrangian function to be

$$
\ell(x, u, \lambda):=f(x)-\int_{I} y_{v}(x) u(d d v)+\int_{J} h_{\beta}(x) \lambda(d / \beta) .
$$

To conform to common practice, we would write condition (a) as

$$
\nabla_{u} \ell\left(x^{*}, u^{*}, \lambda^{*}\right)=l^{\prime}\left(x^{*}, u^{*}, \lambda^{*}\right)=0 .
$$

The following result estallishes circunstances in which this representation is legitimate.

Proposition 2.2 Fix $x \in X$. Let u and $\lambda$ denote totally finite measures on $(I, \mathbf{I})$ and $(J, \mathbf{J})$. Asstume that the expectutions $\bar{g}:=\int_{I} g_{\alpha} u(d \alpha)$ and $\bar{h}:=$ $\int_{J} h_{\beta} \lambda(d \beta)$ both exist. If the st.s of functions $A:=\left\{y_{r}: \alpha \in I\right\}$ and $B$ are each uniformly Lipschitz continuous, the:n $\nabla_{x} \ell(x, u, \lambda)=\ell^{\prime}(x, u, \lambda)$.

Proof: We must establish that

$$
\nabla \int_{I} g_{\alpha}(x) u(d \alpha)=\nabla \bar{g}(x)=\int_{\nabla A(x)} \eta \nabla F(d y)=\int_{I} \nabla g_{c v}(x) u(d \alpha),
$$

where $\nabla F$ is the measure on $X$ induced by $u$. Clearly it suffices to prove that

$$
\langle\eta, \nabla \bar{g}(x)\rangle=\left\langle\eta, \int_{\nabla A(x)} \eta \nabla F(d \eta)\right\rangle \quad \forall \eta \in X .
$$


We note that

$$
\begin{aligned}
\langle\eta, \nabla \bar{g}(x)\rangle & =\bar{g}^{\prime}(x)(\eta) \\
& =\lim _{t \rightarrow(1)} \frac{1}{\epsilon}\{\bar{g}(x+\epsilon \eta)-\bar{g}(x)\} \\
& =\lim _{t \rightarrow 0} \frac{1}{\epsilon}\left\{\int_{I}\left[g_{c}(x+\epsilon \eta)-g_{c r}(x)\right] u(d \alpha)\right\} \\
& =\lim _{t \rightarrow 0} \int_{I} \phi_{t}(x) u(d \alpha)
\end{aligned}
$$

and that

$$
\begin{aligned}
\left\langle\eta, \int_{\nabla A(x)} y \nabla F(d y)\right\rangle & =\int_{\nabla A(x)}\langle\eta, y) \nabla F(d y) \\
& =\int_{I}\left\langle\eta, \nabla g_{c r}(x)\right\rangle u(d \alpha) \\
& =\int_{I} g_{r v}^{\prime}(x)(\eta) u(d \alpha) \\
& =\int_{I t \rightarrow n} \lim _{t} \frac{1}{\epsilon}\left[g_{c r}(x+\epsilon \eta)-y_{c r}(x)\right] u(d \alpha) \\
& =\int_{I} \lim _{t \rightarrow 0} \phi_{f}(\alpha) u(d \alpha) .
\end{aligned}
$$

Since the $g_{\alpha}$ 's are uniformly lipschitz continuous, i.e. $\exists M<\infty$ such that $\left|g_{\alpha}(y)-g_{\alpha x}(z)\right| \leq M\|y-z\| \forall x, y \in X$ and $\forall a \in I$, we have

$$
\left|\phi_{\epsilon}(\alpha)\right| \leq \frac{M}{\epsilon}\|\epsilon \eta\|=M\|\eta\|<\infty .
$$

Then, since $u$ is totally finite, we can apply the Dominated Convergence Theorem to interchange lin1 $t_{-0}$ and $\int_{I}$. This establishes (2); hence, (1). The identical argument establishes that

$$
\nabla \int_{J} h_{\beta}(x) \lambda(d \beta)=\int_{J} \nabla h_{h_{\beta}}(x) \lambda(d \beta)
$$

and the result follows.

We now return to problem (NLP). As in the finite-dimensional case, in order to derive a necessity condition from Proposition 2.1, we must supplement the first-order conditions with a constraint qualification. 
Definition 2.2 Suppose that $x^{*}$ is a feasible point of problem (NLP). We say that $x^{*}$ satisfies the coustraint qualification for problem (NLP) if:

for each nonzero $z \in X$ satis/ying $\left\langle z, \nabla y_{c}\left(x^{*}\right)\right\rangle \geq 0 \forall \alpha \in I_{0}\left(x^{*}\right)$ and $\left\langle z, \nabla h_{\beta}\left(x^{*}\right)\right\rangle=0 \forall \beta \in . I$, there exists $\tau>0$ and a continuous are $C:[0, \tau) \rightarrow X$ satisfying

$$
\begin{aligned}
& C(0)=x^{*}, \\
& C^{\prime}(0)=z, \\
& y_{\alpha}(C(t)) \geq 0 \quad \forall t \in[0, \tau) \text { and } \forall \alpha \in I, \\
& h_{\beta}(C(t))=0 \quad \forall t \in[0, \tau) \text { and } \forall \beta \in . J .
\end{aligned}
$$

Our main result now follows precisely as in the finte-dimensional case.

Theorem 2.1 Let $K=\nabla A_{10}\left(x^{*}\right) \cup \nabla B\left(x^{*}\right)$. Assume

A1: $\quad K$ is compact;

A2: $\quad C(K)$ is closerl.

If $x^{*}$ satisfies the constraint qualification for problem (NLP), then a necessary condition for $x^{*}$ to be a local solution of problem (NLP) is that the first-order conditions hold.

Proof: We invoke Proposition 2.1. Suppose that $x^{*}$ is a local solution and that, $z \in Z_{2}\left(x^{*}\right)$. C'early it must he that $z \neq 0$, so there exists a feasible continuous arc $C:[0, \tau) \rightarrow X$. Since $x^{*}$ is a local solution, for $t>0$ sufficiently small it must be that

$$
f(((1))-f((r(0)) \geq 0
$$

and therefore

$$
\frac{1}{t}[f((\zeta))-f(C(0))] \geq 0
$$

But this implies that

$$
\left[f \circ C^{\prime}\right]^{\prime}(0)=\left\langle\nabla f\left(\left(C^{\prime}(0)\right), C^{\prime}(0)\right\rangle=\left\langle\nabla f\left(x^{*}\right), z\right\rangle \geq 0\right.
$$

which is a contradiction. 


\section{Discussion of Hypotheses}

Let us now examine assumptions Al and A2 of Theorem 2.1. To begin, recall that they antomatically hold in the case of finite programming. Specifically, a finite set is compact, and it is well-known that a finitely generated cone is closed. Hence, assumptions A1 and A2 are exactly the price one nust pay to extend the Farkas lemma approach to necessity conditions from finite programming to infinite proglamming. Of course this extension will be of no value if we cannot find reasonable conditions that imply assumptions $A 1$, A2, and the constraint (unalifiation. Such conditions are the subject of the present section; in the next section, we will present a meaningful example that satisfies our conditions.

We first consider assumption Al, the compactness assumption for the sets $\nabla A_{0}\left(x^{*}\right)$ and $\nabla B\left(x^{*}\right)$. Since polblem (NLP) is stated without reference to these sets, it is obviously cunlsersome to clieck lypotheses involving them. Fortunately, many problems will not recuire this.

Lemma 3.1 Suppose that the $g_{i r}$ and $h_{i j}$ are elements of a real Hilbert space Г. Fix $x \in X$ and let $V_{x}$ denole couluation at a. Suppose that the $g_{\alpha}$ and $h_{\beta}$ are uniformly continuous and that $V_{x}$ is a continuons functional on $A$ and $B$. Assume that the inder sets I and J have been topologized. If I and J are compact and the index maps $\alpha \mapsto y_{c}$ and $\beta \mapsto h_{\beta}$ are continuous, then the sets $\nabla A_{0}(x)$ and $\nabla B(x)$ are compract.

Proof: We argue in terms of the $g_{1}$. Given a sequence $\left\{\alpha_{n}\right\} \subset I_{0}(x)$, we claim that there exists $\sigma_{n} \in I_{0}(x)$ and a subsequence $\left\{\alpha_{n^{\prime}}\right\}$ such that $\nabla g_{\alpha_{n^{\prime}}}(x) \rightarrow \nabla g_{\alpha_{0}}(x)$

The indexing assumptions imply that $A$ is compact. Since $V_{x}$ is continuous on $A$, it follows that the level set $A_{10}(x)=\left\{g_{\alpha}: g_{c x}(x)=0\right\}=$ $\left\{g_{\alpha}: V_{x}\left(g_{\alpha}\right)=0\right\}$ is closed, hence compact itself. Therefore there exists $\alpha_{0} \in I_{0}(x)$ and a subsequence $\left\{\alpha_{n^{\prime}}\right\}$ such that $g_{\alpha_{n^{\prime}}} \rightarrow g_{\alpha_{0}}$.

The convergence indicated is in nom. However, since the $g_{x}$ are uniformly

continuous, the convergence must also be nuiform. But this allows us to write

$$
\lim _{n^{\prime} \rightarrow \infty} \nabla y_{c^{\prime}{ }^{\prime}}(x)=\nabla \lim _{n^{\prime} \rightarrow \infty} y_{e^{\prime}{ }^{\prime}}(x)=\nabla y_{r_{r_{0}}}(x) .
$$


We now derive conditions which imply that assumption A2 holds. We first derive a technical lemma abont axpectations that will be used to show that $K$ compact and $0 \notin C_{1}(K)$ implics $A 2$. This lemma derives from probability theory. An excellent reference for the requisite material is Billingsley [2].

Lemma 3.2 Let $H$ denote "real Hilbert space with inner product $(\cdot, \cdot)$. Let $\mathbf{M}_{1}(K)$ denote the family of probubility measures that concentrate on the set $K \subset H$. If $K$ is compact, then the set of expectations $C_{1}(K):=\left\{\int x \mu(d x):\right.$ $\left.\mu \in \mathbf{M}_{1}(K)\right\}$ is convex and componet.

Remark: As mentioned before, the set, $C_{1}(K)$ is essentially the convex hull of $K$.

Proof: Since $K$ is compact, $\int(y, x) \mu(d x) \leq\|y\| \int\|x\| \mu(d x) \leq\|y\| \sup _{x \in K}\|x\|<$ $\infty$; we are therefore assured that the expectations exist. The convexity of $C_{1}(K)$ follows immediately from the linearity of expectation.

To demonstrate compactuess, consider the sequence $\left\{x_{n}=\int x_{\mu} \mu_{n}(d x)\right.$ : $\left.\mu_{n} \in \mathrm{M}_{1}(K)\right\}$. Since $K$ is compact, $\mathrm{M}_{1}\left(K^{\prime}\right)$ is tight. It follows from Prohorov's Theorem that there exists a weakly convergent subsequence of $\left\{\mu_{n}\right\}$, i.e. that there exists $\mu_{0} \in \mathrm{M}_{1}\left(K^{\prime}\right)$ and a subsequence $\left\{\mu_{n^{\prime}}\right\}$ such that

$$
\int \phi(x) \mu_{n^{\prime}}(d x) \rightarrow \int \phi(x) \mu_{0}(d x)
$$

for all bounded continuons functions $\phi: H \rightarrow(-\infty,+\infty)$. Since $K$ is compact, $(y, \cdot)$ is such a function; hence

$$
\int(y, x) \mu_{n^{\prime}}(d x) \rightarrow \int(y, x) \mu_{0}(d x) \quad \forall y \in H .
$$

Then it must be that the Riesz representers

$$
x_{n^{\prime}}=\int x \mu_{n^{\prime}}(d x) \rightarrow x_{0}:=\int x \mu_{0}(d x),
$$

so the arbitrary sequence $\left\{x_{n}\right\}$ lias a convergent subsequence.

We now remove the restriction that the positive measures used to form expectations liave a total mass of unity.

Lemma 3.3 Let $H$ denote a real Hillure space with inner product $(\cdot, \cdot)$ and origin 0 . Let $\mathrm{M}(K)$ denote the fomily of totally finite, positive measures that concentrate on the set $K \subset H$. If $K$ is compact and $0 \notin C_{1}(K)$, then $C(K):=\left\{\int x \mu(d x): \mu \in \mathrm{M}\left(K^{\prime}\right)\right\}$ is convex and closed. 
Remark: As mentioned before, the set $C(K)$ is essentially the half-cone generated by the convex linll of $K$.

Remark: The conditions that $K$ is compact and $0 \notin C_{1}(K)$ are sufficient but not necessary for the conclusion. To illustrate, let $S$ be a closed subspace of $H$ and let $K \subset S$ be any set such that 0 is an interior point of $C_{1}(K) \subset S$ relative to $S$, e.g. $\{x \in S:\|x\|<1\}$. Then $C(K)=S$ is automatically convex and closed. However, the simple conditions stated in the lemma liave a natural analog in the finite-dimensional theory and are entirely arleculute for the example of Section 4.

Proof: Writing $C(K)=\left\{r: x: x \in C_{1}(K), r=[0,+\infty)\right\}$, it follows from the convexity of $C_{1}(K)$ that $C(K)$ is a convex half-cone. We claim that $C(K)$ is also closed.

Toward that end, suppose that $\left\{y_{n}\right\} \subset C(K)$ with $\left\|y_{n}-\bar{y}\right\| \rightarrow 0$. Write $y_{n}=r_{n} x_{n}$ with $x_{n} \in C_{1}(K)$. By the compactiness of $C_{1}(K),\left\{x_{n}\right\}$ contains a subsequence $\left\{x_{n^{\prime}}\right\}$ with $\left\|x_{n^{\prime}}-\bar{x}\right\| \rightarrow 0$ for some $\bar{x} \in C_{1}(K)$. Moreover, since $0 \notin C_{1}(K),\|\vec{x}\|>0$.

Now let $\epsilon>0$ be arbitrary. By construction, there exists $N(\epsilon)$ such that $n^{\prime} \geq N(\epsilon)$ entails $\left\|x_{n^{\prime}}-\bar{x}\right\| \leq \epsilon$ and $\left\|r_{n^{\prime}} \cdot x_{n^{\prime}}-\bar{y}\right\| \leq \epsilon$. It follows that, if $\epsilon<\|\bar{x}\|$ and $n^{\prime} \geq N(\epsilon)$, then

$$
\frac{\|\bar{y}\|-r}{\|\bar{x}\|+c} \leq r_{n^{\prime}} \leq \frac{\|\bar{y}\|+\epsilon}{\|\bar{x}\|-\epsilon},
$$

so that $r_{n^{\prime}} \rightarrow \bar{r}:=\|\bar{y}\| /\|\bar{x}\|$. Hence,

$$
\begin{aligned}
\left\|y_{n^{\prime}}-\bar{r} \bar{x}\right\| & =\left\|r_{n^{\prime}} \cdot r_{n^{\prime}}-r_{n^{\prime}} \bar{x}+r_{n^{\prime}} \cdot \bar{x}-\bar{r} \cdot \bar{x}\right\| \\
& \leq r_{n^{\prime}}\left\|\cdot r_{n^{\prime}}-\bar{x}\right\|+\left|r_{n^{\prime}}-\bar{r}\right|\|\bar{x}\| \\
& \rightarrow 0 .
\end{aligned}
$$

By the uniqueness of linit.s, $\bar{y}=\bar{r} \cdot \bar{r} \in C\left(K^{\prime}\right)$.

Notice that the hypothesis that $0 \notin C_{1}(K)$ is closely related to the oftimposed (in finite programming) condition of regularity. A feasible point $x^{*}$ is said to be regular if the set. $K$ is linearly independent, i.e. if no finite nonzero linear combination of the constraint gradients at $x^{*}$ can vanish. Our condition is somewhat stronger in one respect, but much weaker in another. On the one hand, we consider arbitrary measures (weights) on $K$, not just 
finitely supported ones. This is analogous to infinite linear combinations, hence stronger; on the other lannl, we only consider probability measures (nonnegative weights totalling unity); this is analogous to convex combinations instead of lineal combinations, lence weaker.

In finite programming, if $x^{*}$ is a regular point, then $x^{*}$ must satisfy the constraint qualification. This pleasant property does not loold in infinite programming; in fact, since the number of linearly independent gradients cannot exceed the dimension of the space $X$, the notion of regularity is wholly inappropriate for the case of semi-infinite programming and somewhat inappropriate in the case of infinite programming. Accordingly, we will search for other conditions that will imply the constraint cualification.

The simplest situation is the one in which all of the constraints are linear. If $x^{*}$ and $z$ are as in Definition 2.2 , then the arc $C(t)=x^{*}+t z$ satisfies

$$
\begin{aligned}
C(0) & =r^{*} ; \\
C^{\prime}(0) & =\because: \\
g_{c *}(C(t)) & =0 \quad \forall t \geq 0, \quad \forall \alpha \in I_{0}\left(x^{*}\right) ; \\
l_{\iota_{\beta}}(C(t)) & =0 \quad \forall t \geq 0, \quad \forall \beta \in . J .
\end{aligned}
$$

Moreover, for each $\alpha \in I \sim I_{0}\left(x^{*}\right)$ (the nonlinding constraints), there exists $\tau(\alpha)>0$ such that

$$
y_{\alpha r}(C(t)) \geq 0 \forall t \in[0, \tau(\alpha)) .
$$

If the number of nonbinding constraints is finite, then we can take $\tau=$ $\inf _{\alpha \alpha}\{\tau(\alpha)\}>0$ and the constraint qualification is automatically satisfied. Otherwise, it may be that inf $f_{n}\{\tau(o)\}=0$ and the constraint qualification may not hold. We are therefore content to establish that the constraint qualification holds for one inportant family of examples.

Both control theory and statistics abonud with constraints of the sort that a function be bounderl by certain values. The following result addresses the prototypical case; we hope that the method of proof will suffice for a variety of applications.

Lemma 3.4 Let $X$ denote a ral llilbert space of functions $x: I \rightarrow(-\infty,+\infty)$. Let $g_{x}$ denote evaluation at $\alpha \in l$. If $X$ is a proper functional Hilbert space, i.e. if the $g_{c}$ are continuous, then the collection of inequality constraints

$$
g_{u}(x)=. r(a) \geq 0 \quad \forall \alpha \in I
$$


satisfies the constraint qualification.

Proof: Since the $g_{\alpha r}$ are continuons and linear, $\nabla g_{\alpha}(x)$ exists $\forall x \in X$. Suppose that $x^{*} \in X$ is a feasilule point and that a nonzero $z \in X$ satisfies

$$
\begin{gathered}
\left\langle z, \nabla g_{c r}\left(x^{*}\right)\right\rangle=g_{c}^{\prime}\left(x^{*}\right)(z)=z(\alpha) \geq 0 \quad \forall \alpha \in I_{0}\left(x^{*}\right), \\
\text { i.e. } \forall a \text { such that } x^{*}(\alpha)=0 .
\end{gathered}
$$

For $t>0$, let

$$
K(t):=\left\{y \in X: x^{*}(\alpha)+t y(\alpha) \geq 0 \quad \forall \alpha \in I\right\} .
$$

We note that the sets $K(t)$ are nested, for suppose that $t_{0}<t_{1}$. If $0 \leq$ $x^{*}(\alpha)+t_{1} y(\alpha) \quad \forall \alpha \in I$, i.e. $y \in K^{\prime}\left(t_{1}\right)$, then

$$
0 \leq \frac{t_{0}}{t_{1}} x^{*}(\alpha)+t_{01} y(\alpha) \leq x^{*}(\alpha)+t_{0, \eta}(\alpha) \quad \forall \alpha \in I
$$

i.e. $y \in K\left(t_{0}\right)$. Thiss, $K\left(t_{1}\right) \subset K\left(t_{0}\right)$.

Suppose that $t_{n} \downarrow t_{0}$. Then $\cup_{n} K\left(t_{n}\right) \subset K\left(t_{0}\right)$; hence, the closure of $\cup_{n} K\left(t_{n}\right)$ is contained in the closure of $K\left(t_{0}\right)$, which is just $K\left(t_{0}\right)$. To establish the converse, let $y_{0} \subset K^{\prime}\left(t_{0}\right)$ and set $y_{n}=\left(t_{0} / t_{n}\right) y_{0}$. Then $y_{n} \subset K\left(t_{n}\right)$ and $\left\|y_{n}-y_{0}\right\| \rightarrow 0$; hence, $K\left(t_{0}\right)$ is contained in the closure of $\cup_{n} K\left(t_{n}\right)$.

Suppose that $t_{n} \uparrow t_{0}$. Then $\cap_{n} K\left(t_{n}\right)$, i.e.

$$
\left\{x^{*}(a)+l_{n !} y(a) \geq 0 \quad \forall a \in I\right\} \quad \forall t_{n} .
$$

Then

$$
\left\{x^{*}(\alpha)+t_{0} \eta(\alpha) \geq 0 \quad \forall \alpha \in I\right\}
$$

i.e. $y \in K\left(t_{0}\right)$.

It now follows from Proposition 3.22 in Attonch [1] that, if $t_{n} \rightarrow t_{0}$, then $K\left(t_{n}\right) \rightarrow K\left(t_{0}\right)$ in the souse of Mosco-convergence of closed convex sets. Furthermore, Proposition 3.34 in Attouch [1] states that the Moscoconvergence of closed convex sets is equivalent to the convergence of the projections of an arbitrary point into these sets. Therefore, let $z^{*}(t)$ denote the projection of $Z$ into $K(t)$. 'Then, $t_{n} \rightarrow t_{0}$ entails $\left\|z^{*}\left(t_{n}\right)-z^{*}\left(t_{0}\right)\right\| \rightarrow 0$, and we conclude that $z^{*}(t)$ is a continuons are for $t>0$. Moreover, since $x^{*}(\alpha)=0$ entails $z(\alpha) \geq 0, z$ is contained in the closine of $\cup_{t>0} K(t)$. We can therefore close the arc by setting $z^{*}(0)=z$. 
Now let $C(t):=x^{*}+t z^{*}(t)$. By construction, $C$ is a feasible continuous arc with $C(0)=x^{*}$. Moreover,

$$
\lim _{t \rightarrow 0} \frac{1}{t}\|C(t)-C(0)-t z\|=\lim _{t \rightarrow 0} \frac{1}{t}\left\|t z^{*}(t)-t z\right\|=\lim _{t \rightarrow 0}\left\|z^{*}(t)-z\right\|=0,
$$

so $C^{\prime}(0)=z$. This verifies the conditions specified by Definition 2.2 .

Remark: It is also possible to give an elementary proof that the arc $z^{*}(t)$ is continuous. The use of Mosco-convergence was suggested to us anonymously by the referee. The equivalence of Mosco-convergence and the convergence of the projections of an arbitrary point is due to Sonntag [18].

\section{An Example}

We now apply our resints to oldian necessity conditions for a well-known problem from the statistical literature on probalility density estimation. Watson and Leadbetter [24] songlit to mininize the mean integrated squared error of a kermel probalility density estimator. Specifically, given independent and identically distributed ranclon variables $X_{1}, \ldots, X_{n}$ with probability density function $\delta$, they analyzer the optimization problem

$$
\underset{K_{n} \in L^{2}(-\infty,+\infty)}{\operatorname{minimize}} E \int_{-\infty}^{\infty}\left[\frac{1}{n} \sum_{i=1}^{n} K_{n}\left(x-X_{i}\right)-\delta(x)\right]^{2} d x
$$

It turns out that solutions are' typically not everywhere nonnegative, which results in estimates that are not thomselves probaloility densities. This is a matter of taste, but if we prefer to estinate densities with densities, then we must confront the constrained optimization problem

$$
\begin{array}{ll}
\underset{K_{n} \in L^{2}(-\infty,+\infty)}{\operatorname{mininize}} & E \int_{-\infty}^{\infty}\left[\frac{1}{n} \sum_{i=1}^{n} K_{n}\left(x-X_{i}\right)-\delta(x)\right]^{2} d x \\
\text { subject to } & K_{n}(x) \geq 0 \quad x \in(-\infty,+\infty) \\
& \int_{-\infty}^{\infty} K_{n}(x) d x=1 .
\end{array}
$$

This problem does not yield to variational nethods, making it a natural candidate for the application of om multiplier theory. We proceed to formulate it in that context. 
Consider the Sobolev spare $H^{1}\left[\alpha_{1}, \alpha_{2}\right]$, which is defined by endowing the vector space

$$
\left\{x: x^{(i)} \in L^{2}\left[\alpha_{1}, \alpha_{2}\right] \text { for } j=0,1\right\}
$$

with the inner product

$$
\langle x, y\rangle=\sum_{i=11}^{1}\left\langle r^{(j)}, y^{(j)}\right\rangle_{L^{2}\left[\alpha_{1}, c_{2}\right]} .
$$

It should be noted that the derivatives in the definition of $H^{1}\left[\alpha_{1}, \alpha_{2}\right]$ are taken in the sense of distributions. It is woll known that $H^{1}\left[\alpha_{1}, \alpha_{2}\right]$ is a proper functional Hilbert space and that each element of $H^{1}\left[\alpha_{1}, \alpha_{2}\right]$ is absolutely continuous. See Appendix I of Tapia and Thompson [20] for a cliscussion of the analogous Sobolev space, $H^{1}(-\infty,+\infty)$. Notice that, if $\delta \in H^{1}(-\infty,+\infty)$, then the restriction of $\delta$ to $\left[\alpha_{1}, \alpha_{2}\right]$ is an element of $H^{1}\left[\alpha_{1}, \alpha_{2}\right]$.

We now return to the problem of Watson and Leadbetter, which we reformulate as problem (WL):

$$
\begin{aligned}
& \underset{x_{n} \in X}{\operatorname{minimize}} \quad f\left(x_{n}\right)=E \int_{-\infty}^{\infty}\left[\frac{1}{n} \sum_{i=1}^{n} \bar{x}_{n}\left(\alpha-\chi_{i}\right)-\delta(\alpha)\right]^{2} d \alpha \\
& \text { subject to } \quad y_{n}\left(a_{n}\right)=x_{n}(\alpha) \geq 0 \forall \alpha \in I \\
& h\left(x_{n}\right)=\int_{I} u_{n}(\alpha) d \alpha-1=0 \text {, }
\end{aligned}
$$

where $I=\left[\alpha_{1}, \alpha_{2}\right], X=H^{1}\left[\alpha_{1}, \gamma_{2}\right]$, and $\bar{x}_{n}$ denotes the extension of $x_{n}$ to $(-\infty,+\infty)$ defined by $\bar{x}_{n}(\alpha)=0$ il $\alpha \notin I$; and where the expectation is taken with respect to the independent and irlentically distributed random variables $\chi_{i}, i=1, \ldots, n$, having polability ansity function $\delta \in H^{\mathbf{1}}(-\infty,+\infty)$. We have modified the original pollem in two ways. First, we have demanded some additional smoothness. Serond, we lave restricted attention to kernels supported on $\left[\alpha_{1}, \alpha_{2}\right]$. We proces to verify that Theorem 2.1 can be applied to problem (WL).

The point evaluation functionals $y_{c} \in \Gamma=X^{*}$ are both linear and continuous, hence continuously differentialble and also uniformly continuous. It is also easily checked that $f, h \in C^{1}(X)$. Furthermore, the set $\nabla B(x)=\{\nabla h(x)\}$ is obvionsly compact. We also have

Lemma 4.1 For problem (WL), llhe set $\nabla A(x)=\left\{\nabla y_{c r}(x): \alpha \in I\right\}$ is compact. 
Proof: We apply Lemma 3.1. The point evaluation functionals $V_{x} \in \Gamma^{*}$ are continuous, since $V_{x}\left(y_{c}\right)=y_{w}(x)=x(\alpha)$. Since $I$ is compact, it remains only to demonstrate that the index map $\alpha \mapsto g_{c r}$ is contimuous.

Consider the optimization problem

$$
\begin{array}{ll}
\operatorname{minimize} & \int_{u_{1}}^{a_{2}}\left[x^{\prime}(\alpha)\right]^{2} d \alpha \\
\text { suljject to } & x\left(a_{1}\right)=b_{1}, x\left(a_{2}\right)=b_{2} .
\end{array}
$$

It is a trivial exercise in the calculus of variations to establish that the minimizer is a straight line witll slope

$$
a^{\prime}(\alpha)=\left(b_{2}-b_{1}\right) /\left(a_{2}-a_{1}\right) .
$$

This yields a minimunn objective function value of $\left|b_{2}-b_{1}\right|^{2} /\left|a_{2}-a_{1}\right|$. It follows that any $x \in X$ with $x\left(a_{1}\right)=b_{1}$ and $x\left(a_{2}\right)=b_{2}$ must satisfy

$$
\|x\|^{2} \geq\left|b_{2}-b_{1}\right|^{2} /\left|a_{2}-a_{1}\right| .
$$

Now suppose that $\alpha_{n} \rightarrow n_{0}$ as $n \rightarrow \infty$. Then (3) allows us to write

$$
\begin{aligned}
\left\|g_{\alpha_{n}}-g_{\alpha_{0}}\right\| & =\operatorname{sip}_{\|x\| \leq 1}\left|g_{\alpha_{r_{n}}}(x)-g_{\alpha_{0}}(x)\right|=\sup _{\|x\| \leq 1}\left|x\left(\alpha_{n}\right)-x\left(\alpha_{0}\right)\right| \\
& \leq \sup _{\|x\| \leq 1} \mid \alpha_{n}-\left(\left.\alpha_{0}\right|^{\frac{1}{2}}\|x\|=\left|\alpha_{n}-\alpha_{0}\right|^{\frac{1}{2}} \rightarrow 0 \text { as } n \rightarrow \infty .\right.
\end{aligned}
$$

Next, we show that our conditions on $K$ loold.

Lemma 4.2 For problem (WL), let $K=\nabla A(x) \cup \nabla h(x)$. Then $C_{1}(K)$ does not contain the origin of $X=H^{1}\left[\sigma_{1}, \alpha_{2}\right]$.

Proof: We exploit the fact that the gradient is the Riesz representer of the directional derivative. Let $\eta \in X$; the'm

$$
\begin{aligned}
y_{(r)}^{\prime}(x)(\eta) & =\lim _{t \rightarrow 1} \frac{1}{t}\left[g_{c r}\left(x+t_{\eta}\right)-y_{c r}(x)\right] \\
& =\lim _{t \rightarrow 1)} \frac{1}{t}[x(\alpha)+t \eta(\alpha)-x(\alpha)] \\
& =\eta(\alpha)
\end{aligned}
$$


and

$$
\begin{aligned}
h^{\prime}(x)(\eta) & =\lim _{t \rightarrow 0} \frac{1}{t}[h(x+t \eta)-h(x)] \\
& =\lim _{t \rightarrow 0} \frac{1}{t}\left[\int_{l}[x+t \eta](\alpha) d \alpha-1-\int_{I} x(\alpha) d \alpha+1\right] \\
& =\int_{I} \eta(\alpha) d \alpha .
\end{aligned}
$$

Hence, $\nabla g_{\alpha}(x)$ musst satisfy

$$
\langle\nabla ! y(x), \eta\rangle=\eta((r) \quad \forall \eta \in X
$$

and $\nabla h(x)$ must satisfy

$$
\langle\nabla h(x), \eta\rangle=\int_{I} \eta(\alpha) d r \quad \forall \eta \in X
$$

Now suppose that there exists $\mu \in \mathbf{M}_{1}(K)$ such that $\int_{K} y \mu(d y)=0$. Let $\lambda=\mu(\nabla h(x))$ and let $(1-\lambda) u$ denote the measure on $(I, \mathbf{I})$ induced by $\mu$. Then it must be that, $\forall \eta \in X$,

$$
\begin{aligned}
0 & =\langle l, \eta\rangle \\
& =\left\langle\int_{K} y \mu(d y) \cdot \eta\right\rangle \\
& =\int_{K}\langle y, \eta\rangle \mu(d y) \\
& =\int_{\nabla \cdot d(x)}\langle y, \eta\rangle \mu(d y)+\lambda\langle\nabla h(x), \eta\rangle \\
& =\int_{\nabla A(x)} \eta(\alpha) \mu(d y)+\lambda \int_{I} \eta(\alpha) d \alpha \\
& =(1-\lambda) \int_{I} \eta(\alpha) u(d \alpha)+\lambda \int_{I} \eta(\alpha) d \alpha .
\end{aligned}
$$

But the last expression in (4) is strictly positive if $\eta \in X$ is strictly positive on $I$; hence, $C_{1}(K)$ camnot contain the origin of $X$.

Remark: If $u$ is a finitely supported signed measure, say $u=\sum_{i=1}^{m} u_{i} \mathbf{1}\left(\alpha_{i}\right)$, where 1 denotes point-mass, then (4) reduces to

$$
0=(1-\lambda) \sum_{i=1}^{m} u_{i} \eta\left(\alpha_{i}\right)+\lambda \int_{I} \eta(\alpha) d \alpha .
$$


If $\lambda=1$, this equality fails for (say) $\eta(\alpha) \equiv 1$; if $\lambda \neq 1$, this equality fails for any $\eta$ satisfying $\eta\left(\alpha_{i}\right)=-u_{i}$ and $\int_{l} \eta(\alpha) d \alpha=0$. Thus, the condition of regularity also holds for problen (WL). Notice, however, that the restriction to finite linear combinations in the definition of linear independence is crucial to this conclusion. If arbitrary signed measures are allowed, then take $u$ to be the negative uniform measure on $I$ and put $\lambda=1 /\left(\alpha_{2}-\alpha_{1}+1\right)$. Then the last expression in (4) is

$$
\begin{aligned}
& (1-\lambda) \int_{I} \eta(\alpha) u(d \alpha)+\lambda \int_{I} \eta(\alpha) d \alpha \\
= & {\left[1-\frac{1}{\alpha_{2}-\alpha_{1}+1}\right] \frac{1}{\alpha_{2}-\alpha_{1}} \int_{1} \eta(\alpha) d \alpha+\frac{1}{\alpha_{2}-\alpha_{1}+1} \int_{I} \eta(\alpha) d \alpha, }
\end{aligned}
$$

which does indeed vanish $\forall \eta \in X$. This distinction shonld not be surprising. Roughly stated, finitely many values do not determine a function's Lebesgue integral, but all values together do.

Finally, the equality constrint in problem (WL) is easily incorporated into the proof of Lemma 3.4. This provides a means of verifying that any feasible point for problem (WL) satisfies the constraint qualification. Theorem 2.1 therefore applies: a neressary condition for $x_{n}^{*}$ to be a local solution of problem (WL) is that the first-order conditions hold.

Let us make some funther olservations concerning problem (WL). The objective function is strictly convex and the constraint set is convex. It follows that any local solution will be the nuique global solution. It is well known that the variational inerpulity which serves as a necessity condition when the constraint set is convex serves as a sufficiency condition when the objective function is also convex. A rather straightforward argunent can be used to show that, in the case of a convex constraint set, condition (i) of Proposition 2.1 , namely $Z_{2}\left(x^{*}\right)=\phi$, inplies the variational inequality necessity condition. These comments say that, in the case of a convex program where the constraint qualification holds (as is the case for problem (WL)), the existence of Lagrange 1n11/iplicrs (Proposition 2.1) is both necessary and sufficient for $x^{*}$ to be a global minimizer.

Our theory, the above comments and some straightforward computations lead us to the following result concerning problem (WL): $x_{n}^{*}$ is the unique global minimizer if and only il there exists a totally finite measure concentrating on $\left[\alpha_{1}, \alpha_{2}\right]$, with density function $u_{n}^{*}$, and a real number $\lambda_{n}^{*}$, such that 
(a) $u_{n}^{*}(\alpha)=2 \frac{n-1}{n}\left[x_{n}^{*} * \delta * \bar{\delta}\right](\alpha)-2[\delta * \bar{\delta}](\alpha)+\frac{2}{n} x_{n}^{*}(\alpha)+\lambda_{n}^{*} \quad \forall \alpha \in\left[\alpha_{1}, \alpha_{2}\right]$,

(b) $x_{n}^{*}(\alpha) \geq 0 \quad \forall \alpha \in\left[\gamma_{1}, \alpha_{2}\right]$,

(c) $\int_{\alpha_{1}}^{\alpha_{2}} x_{n}^{*}(\alpha) d \alpha=1$

(d) $x_{n}^{*}(\alpha) u_{n}^{*}(\alpha)=0 \quad \forall \alpha \in\left[\alpha_{1}, \alpha_{2}\right]$,

(e) $u_{n}^{*}(\alpha) \geq 0 \quad \forall \alpha \in\left[\alpha_{1}, \alpha_{2}\right]$.

In condition $(\mathrm{a}), \bar{\delta}(\alpha):=\delta(-\alpha)$, and $*$ denotes convolution.

Since problem (WL) is highly nontrivial, it is not surprising that the corresponding necessity conditions are somewhat complicated. A more detailed analysis of these conditions was nudertaken by Trosset [21]. Nevertheless, it is evident from the material presented here that the theory developed in Sections 2 and 3 can be productively applied to a body of problems admitting an infinite programming formulation.

\section{Acknowledgements}

We gratefully and sincerely thank the late Magnus Hestenes, William Karush, and Frederick Valentine for so gencronsly sharing their recollections of a beautiful and exciting era in 20th century mathenatios in which they participated. These discussions were a rare privilege, and they significantly enhanced both our understanding and onr enjoyment of onr own research.

We also thank an anonymons referee for comments and suggestions that led to a more elegant presentation of the theory. Finally we thank Stephen Robinson for leading us to the work of Prékopai[17].

\section{References}

[1] H. Attonch. Variational Contergence for Functions and Operators. Pitman, Boston, 1984.

[2] P. Billingsley. Conotryende of Probubility Measures. John Wiley and Sons, New York, 1968.

[3] G. A. Bliss. Normality and abmormality in the calculus of variations. Transactions of the Ameriorn Mathematical Society, 43:365-376, 1938. 
[4] J. Dieudonné. Sur le théorène de Hahn-Banach. Rev. Sci., 79:642-643, 1941 .

[5] J. Farkas. Über die Theorie de1 einfachen Ungleichungen. Journal für die reine und angewandte: Mathematik, 124:1-27, 1901.

[6] A. V. Fiacco and C. P. Mc('ormick. Nonlinear Programming: Sequential Unconstrained Minimization Tecluniques. Joln Wiley and Sons, New York, 1968.

[7] I. V. Girsanov. Lectures on Mathematical Theory of Extremum Problems. Springer-Verlag, New York, 1972.

[8] A. Coberna, M. A. López, and .J. Pastor. Farkas-Minkowski systems in semi-infinite programming. Applied Mathematics and Optimization, $7: 295-308,1981$.

[9] M. Guignard. Generalized Kulun-Tucker conditions for mathematical programming problems in a. Banach space. SIAM Journal on Control, $2: 232-241,1969$.

[10] M. R. Hestenes. Optimization Throry: The: Finite Dimensional Case. Jolnn Wiley and Sons, Now York, 1975.

[11] F. John. Extremmu pohlems with inegualities as subsidiary conditions. In K. O. Friedrichs, O. E. Nengebuner, and J. J. Stoker, editors, Studies and Essays Presented to R. Courant on His 60th Birthday, pages $187-$ 204, Interscience Publishors, New York, 1948.

[12] W. Karush. Minima of Functions of Seneral Variables with Inequalities as Side Conditions. Master's thesis, University of Chicago, 1939.

[13] H. W. Kulhn. Nonlinear programming: a historical view. In R. W. Cottle and C. E. Lemke, editors, Nonlinem Programming, pages 1-26, SIAMAMS, American Mathemalical Society, Providence, 1976. An appendix summarizes Karush's master's thesis.

[14] H. W. Kulun and A. W. Tucker. Nonlinear programming. In .J. Neyman, editor, Proceedings of the Serond Betkeley Symposium on Mathematical Statistics and Probability pages 481-492, University of California Press, Berkeley, 1951. 
[15] A. Mayer. Begrüdung der Lagrange'schen Multiplicatorenmethode in der Variationsredhnung. Matliematische: Annalen, 26:74-82, 1886.

[16] K. R. Parthasarathy. Probubility Measures on Metric Spaces. Academic Press, New York, 1967.

[17] A. Prékopa. On the developnnent of optimization theory. American Mathematical Monthly, 87:527-542, 1980.

[18] Y. Sonntag. Interprétation géométrique de la convergence d'une suite de convexes. C. R. Arad. Sic Parts, Sérte A, 282:1099-1100, 1976.

[19] A. Takayama. Mathemaliral Eronomics. Dryden Press, Hinsdale, IL, 1974.

[20] R. A. Tapia and J. R. Thompson. Nonparametric Probubility Density Estimation. Johns Hopkins University Press, Baltimore, 1978.

[21] M. W. Trosset. Optimal shapes for kernel density estimation. Communications in Statistics - Thtory and Methods, 22:375-391, 1993.

[22] F. A. Valentine. Conver S'ts. Krieger, New York, 1964. Reprinted with corrections in 1976.

[23] F. A. Valentine. The prohlen of Lagrange with differential inequalities as added side conditions. In Contributions to the Calculus of Variations, 1933-1937, pages 403-447, Nuiversity of Chicago Press, Chicago, 1937. Ph.D. thesis, Jniversity of (hirago.

[24] G. S. Watson and M. R. Learlleetter. On the estimation of the probability density, I. Annals of Malhrmalical Statistics, 34:480-491, 1963. 